\title{
Cost implications \& Clinical Prudence in Managing Chronic Coronary Syndromes post ESC-Guidelines
}

David KL Quek, FRCP, FAsCC, FACC, FESC

Stable angina or stable coronary artery disease has now been redefined as chronic coronary syndromes (CCS) partly to be more inclusive for several hitherto under-represented, under-recognised non-obstructive coronary artery entities that present as angina-like or myocardial ischemic syndromes. ${ }^{1}$

However, with the adoption of this wider interpretation, came more diagnostic investigations and algorithms that make the management of this CCS, far more complex and possibly much more intrusive and resource-demanding. Figure 1 demonstrates the diagnostic pathway now recommended for angina and suspected coronary artery diesase (CAD), by the European Society of Cardiology.

This suggested clinical pathway particularly the advocacy for early coronary CT angiography and more specialised imaging to determine likelihood for obstructive CAD, is especially resourceand expertise-demanding for hardpressed economies that rely on fee-for-service healthcare services, but also for single payer national healthcare schemes. This is particularly so in lowereconomy nations in Asia and Africa, where healthcare costs have been rising upwards of $7 \%$ per year (Table 1). ${ }^{2}$

Healthcare inflation rates (Table 1) are projected to be $12.6 \%$ for Malaysia, $11 \%$ for Indonesia, $12 \%$ for India, 9.3\% for Singapore, 9.2\% for Thailand, $12.1 \%$ for Vietnam, 8.2\% for Philippines, 9.8\% for China. For more established economies with state-(single)payer national healthcare systems, the inflation rates are lower: Austalia 3.2\%, New Zealand 6.0\%, South Korea 4.0\%, Taiwan $6.3 \%$.

As we adopt the newer standard of care for our CCS patients, it is expected that cost-escalation as well as resource-utilisation would become stress point challenges for finite healthcare resources. These added diagnostic investigations remain controversial as they have yet to be proved cost-effective or prudent, or even clinically necessary or beneficial.

\section{Figure 1}

Approach for the initial diagnostic management of patients with angina and suspected coronary artery disease.

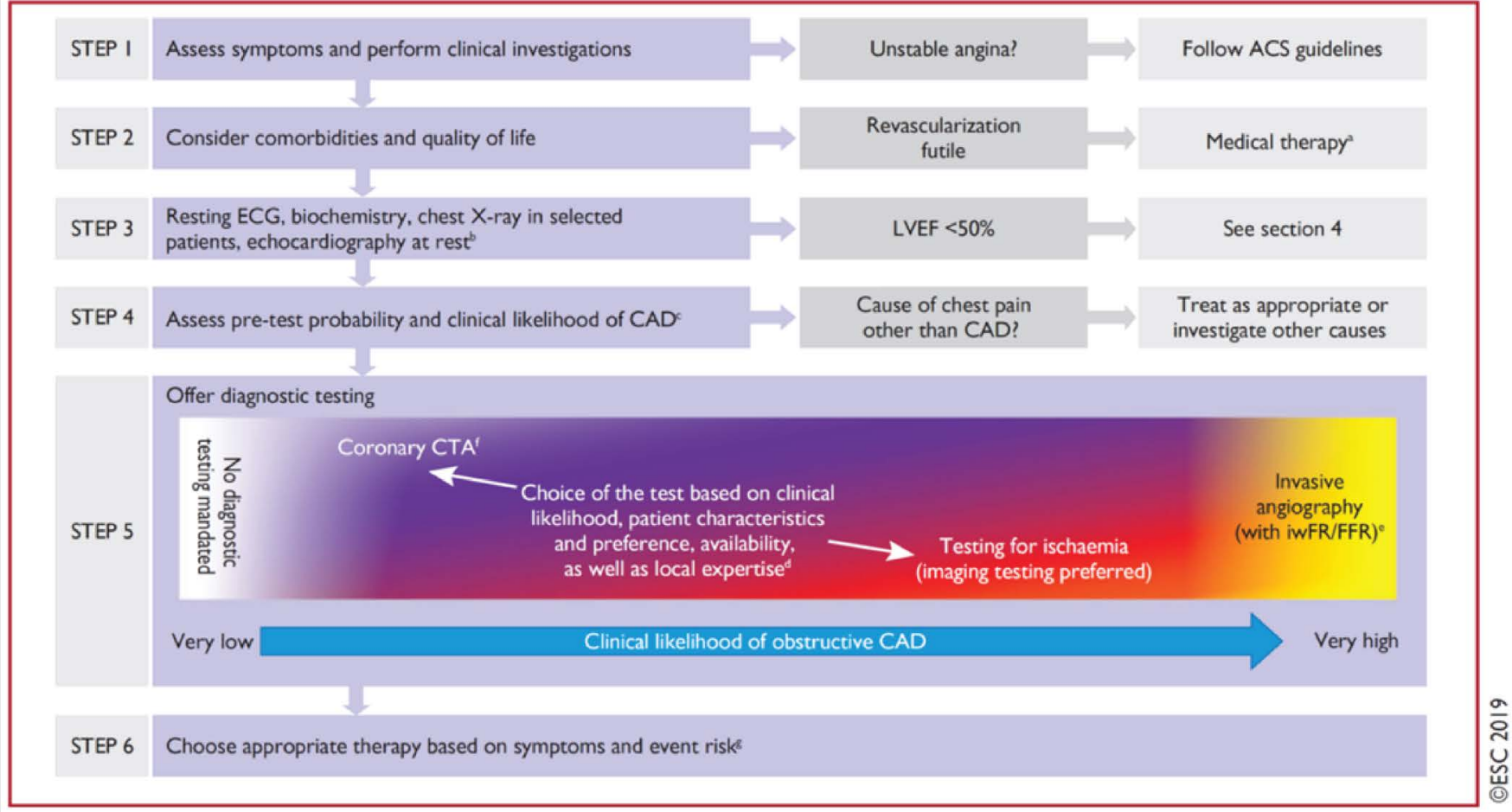


Table 1

\section{Global average medical trend rates by country, 2018-2020}

\begin{tabular}{|c|c|c|c|c|c|c|}
\hline & \multicolumn{3}{|c|}{ Gross cost trend } & \multicolumn{3}{|c|}{ Net cost trend (net of general inflation) } \\
\hline & 2018 & 2019 & $2020^{\ddagger}$ & 2018 & 2019 & $2020^{:}$ \\
\hline Globalt & 6.5 & 6.7 & 6.8 & 4.3 & 4.5 & 4.5 \\
\hline Asia Pacific & 6.8 & 7.1 & 7.1 & 5.1 & 5.2 & 5.1 \\
\hline Australia & 3.4 & 3.7 & 3.2 & 1.4 & 1.6 & 0.9 \\
\hline China* & 9.2 & 10.1 & 9.8 & 7.1 & 7.8 & 7.3 \\
\hline Hong Kong* & 7.5 & 8.2 & 8.3 & 5.1 & 5.8 & 5.8 \\
\hline India & 11.0 & 11.3 & 12.0 & 7.5 & 7.5 & 7.8 \\
\hline Indonesia & 9.0 & 9.8 & 11.0 & 5.8 & 6.4 & 7.4 \\
\hline Malaysia* & 11.9 & 11.6 & 12.6 & 11.0 & 9.6 & 10.1 \\
\hline New Zealand & 7.0 & 7.5 & 6.0 & 5.4 & 5.5 & 4.1 \\
\hline Philippines* & 5.3 & 7.9 & 8.2 & 0.1 & 4.0 & 4.9 \\
\hline Singapore & 8.8 & 8.3 & 9.3 & 8.3 & 6.9 & 7.9 \\
\hline South Korea & 3.8 & 4.0 & 4.0 & 2.3 & 2.6 & 2.5 \\
\hline Taiwan & 6.0 & 6.1 & 6.3 & 4.5 & 5.0 & 5.0 \\
\hline Thailand* & 8.1 & 9.0 & 9.2 & 7.0 & 8.0 & 8.0 \\
\hline$V_{i e t n a m}^{*}$ & 9.7 & 10.9 & 12.1 & 6.2 & 7.8 & 8.8 \\
\hline
\end{tabular}

Willis Towers Watson. 2020 Global medical trends survey report

For most Asian cardiac catheterization laboratories, which are already limited in number and access, physiological testing such as fractional flow-reserve testing is frequently too expensive to be utilized routinely. This is also true of intravascular imaging such as IVUS or OCT. With the position of the ESC guidelines advocating best practices for these to be used more routinely, most healthcare services would face even more severe financial as well as specialist constraints.

So how do we address these real-world inequities? How do we decide which patient to investigate more, and which to defer safely, by offering less precise but optimized medical therapy first? Should we be more selective and prudent and perhaps find some equipoise in routine utilization pressures vs. commonsense reasonable access considerations?

Yosikawa and Kimura ${ }^{3}$ has recently posed this question of equipoise in decision-making for percutaneous coronary intervention in stable CAD. Here, they argued that careful use of FFR (fractional flow reserve, invasive) testing to determine benefits for intervention, outweighs medical therapy alone, based largely on the meta-analysis of Zimmermann et al ${ }^{4}$ comparing the combined studies of FAME-2, DANAMI-3-PRIMULTI and Compare-Acute (see Figure 2). By this reckoning, this analysis would mean greater push for FFR testing with cardiac catheterization to routinely investigate chest pain syndromes.

Figure 2

Landmark analyses of primary composite endpoint and its components.

The hazard ratios of the primary composite outcome of cardiac death or myocardial infarction and of components of the primary composite outcome shown according to the time from randomization (7 days or less vs. 8 days or more).

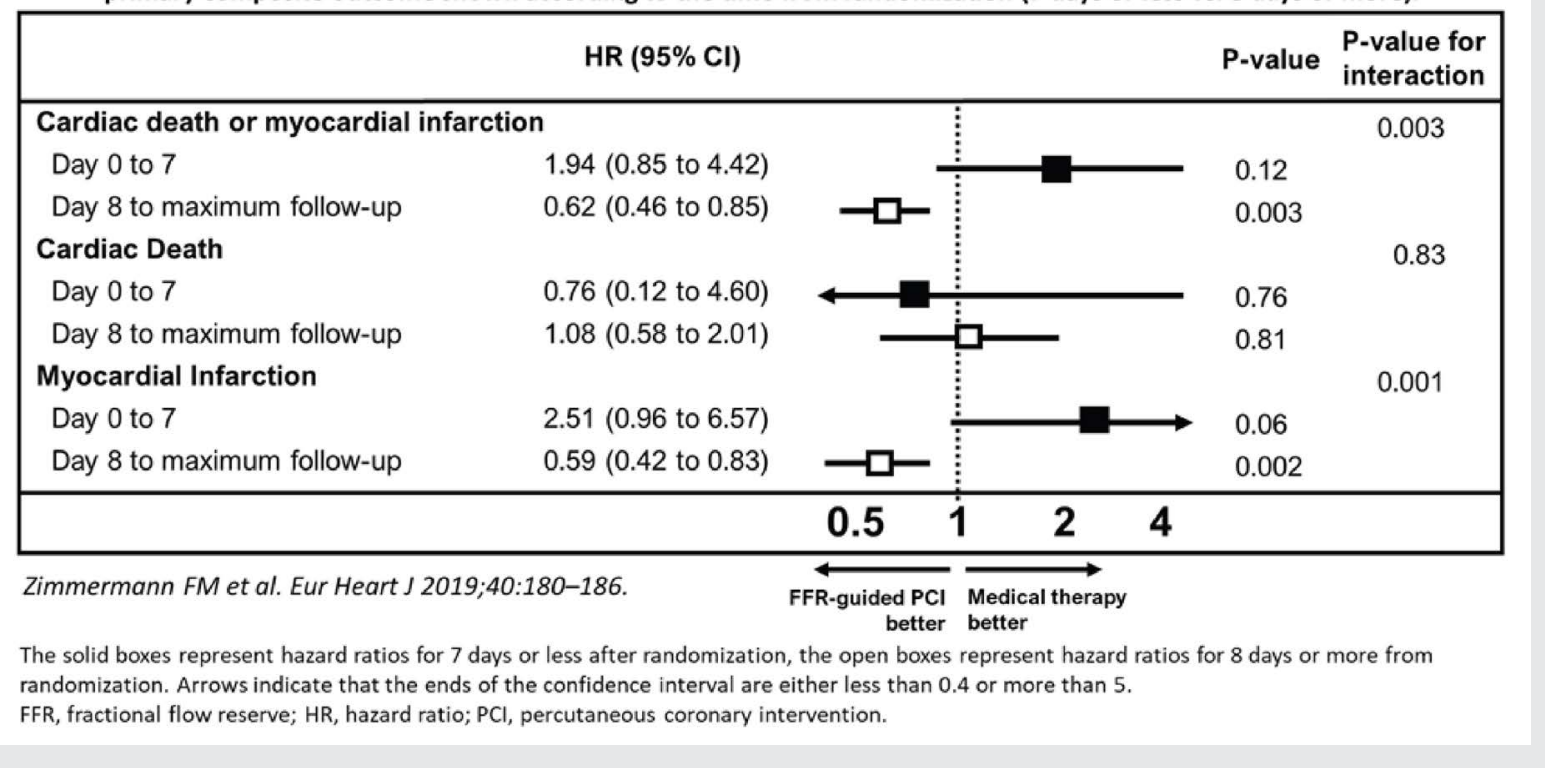


But should this be the conclusion? When you scrutinize the data and results however, the combined benefits were driven predominantly by later reduction of myocardial infarctions by $41 \%(p=0.002)$. There was no significant effect on total mortality i.e. there was no difference with medical therapy alone. Interestingly, the risk of $\mathrm{Ml}$ in the first week following $\mathrm{PCl}$ was some 2.5 times more $(p=0.06)$ when compared with OMT.

From the health economist and health insurer's point of view, however, many $(73 \%)$ of the insurance-claimed medical investigations have been construed as 'overuse of care due to medical practitioners recommending too many services', with over $50 \%$ of the healthcare insurance claims coming from cardiovascular (circulatory system) disorders (see Figure 3). Therefore, as physicians we owe it to our patients as well as our health system to find equipoise in how we manage our angina

patients in this modern era of greater and greater dependency on wider testing protocols, adopting of new technology, new devices, and therapies to offer our patients. Finding the sweet spot of best cost-benefit efficiencies should be our remit.

Without going into the specifics or polemics of insurer defaultreliance on extreme cost-benefit analysis, as physicians we have to address the mounting costs of cardiovascular care that have become one of the main determinants in healthcare cost equation.

To complicate further, a recent 2020 review of chest pain syndromes from Canada of over 1.4 million patients, found benefits from more rigorous non-invasive testing, with about $25 \%$ reduction in major adverse cardiovascular events. ${ }^{5} 1.17$ million received no testing while some 317,056 underwent noninvasive testing. This cohort was followed up for a mean

\section{Figure 3}

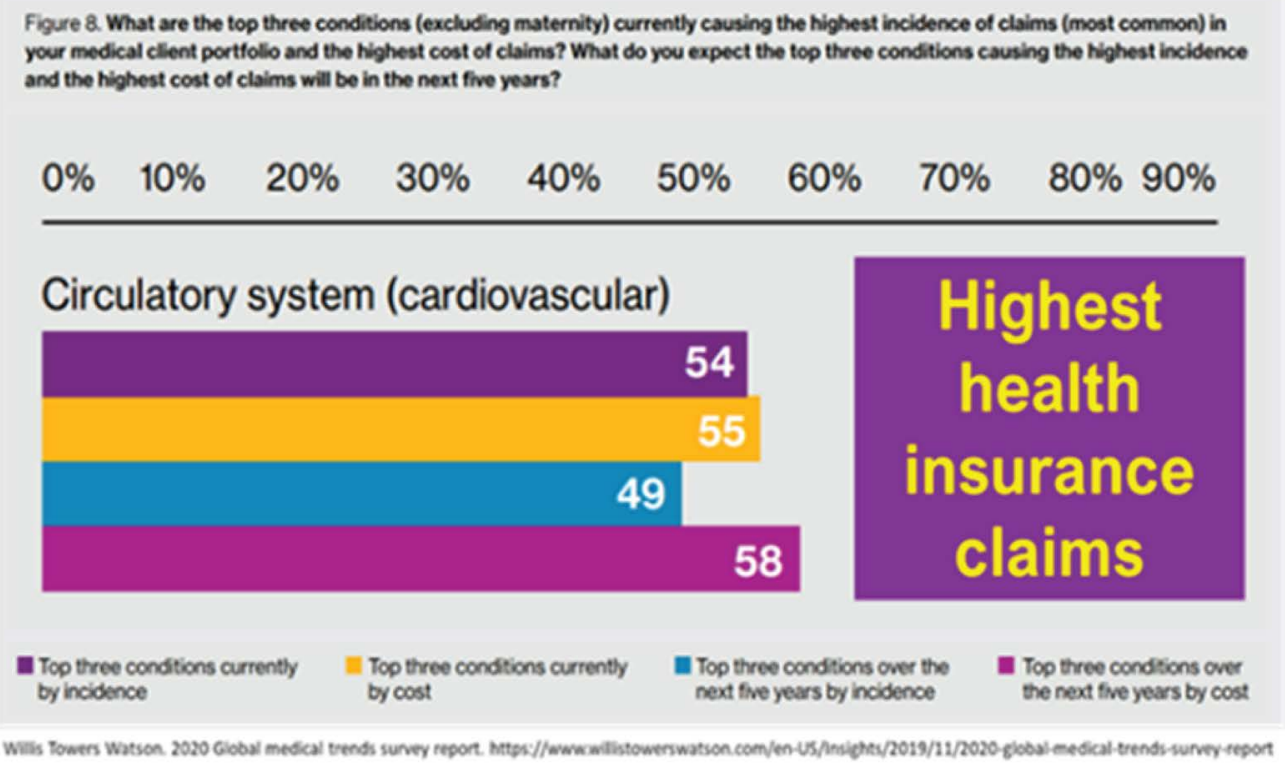
of 4.1 years. Among those not tested, 26,756 $(\approx 1.5 \%)$ underwent invasive coronary angiography within the 90-day landmark period. During 5 years follow-up, downstream invasive angiography (3.8\% versus $4.0 \%, P=0.01)$ and revascularization $(1.5 \%$ versus $1.7 \%, P<0.01)$ via percutaneous coronary intervention or coronary artery bypass grafting, were statistically lower in those who had noninvasive testing (NIT) compared with those who had not undergone testing.

Roifman et al, ${ }^{5}$ showed that the benefits of testing were consistent

\section{Table 2}

\section{Unadjusted Outcomes Comparing the NIT and No-Testing Groups}

\begin{tabular}{|l|c|c|}
\hline & No Testing & Noninvasive Testing \\
\hline Invasive angiography & $46666(4.0 \%)$ & $11950(3.8 \%)$ \\
\hline $\begin{array}{l}\text { Coronary revascularization } \\
(\%)\end{array}$ & $19569(1.7 \%)$ & $4833(1.5 \%)$ \\
\hline $\begin{array}{l}\text { Unstable angina, acute } \\
\text { myocardial infarction, or } \\
\text { cardiovascular mortality (\%) }\end{array}$ & $32853(2.8 \%)$ & $5428(1.7 \%)$ \\
\hline $\begin{array}{l}\text { Acute myocardial infarction } \\
(\%)\end{array}$ & $15021(1.3 \%)$ & $2979(0.9 \%)$ \\
\hline Unstable angina (\%) & $5276(0.5 \%)$ & $1123(0.4 \%)$ \\
\hline Cardiovascular mortality (\%) & $15941(1.4 \%)$ & $1829(0.6 \%)$ \\
\hline
\end{tabular}

NIT indicates noninvasive diagnostic tests. Roifman let al. J Am Heart Assoc. 2020;9:e015724. for all 3 constituents of the composite endpoints (see Table 2, Figure 4). There were significantly fewer unstable angina (HR, 0.87; 95\% Cl, 0.82-0.93 for the noninvasive diagnostic testing, NIT, versus the no-testing group), myocardial infarction (HR, 0.83; 95\% Cl, 0.79-0.86 for the NIT versus the no-testing group) and cardiovascular mortality (HR, 0.68; $95 \% \mathrm{Cl}, 0.65-0.72$ for the NIT versus the no-testing group).

So, it appears that in the best-case scenarios, chest pain syndromes should be assessed accurately with as appropriate tests as possible, if cost is not a concern, because these would offer the best long-term outcomes for patients with symptoms. Some $20 \%$ of these patients would fare better clinically, when they undergo appropriate NIT. The odds-ratio benefits in terms of generating 
Figure 4

\section{Major adverse cardiovascular events compared between the NIT and no-testing groups in the overall cohort, in patients $<65$ years of age, in patients aged 65 years and older, and stratified by different physician diagnostic codes.}

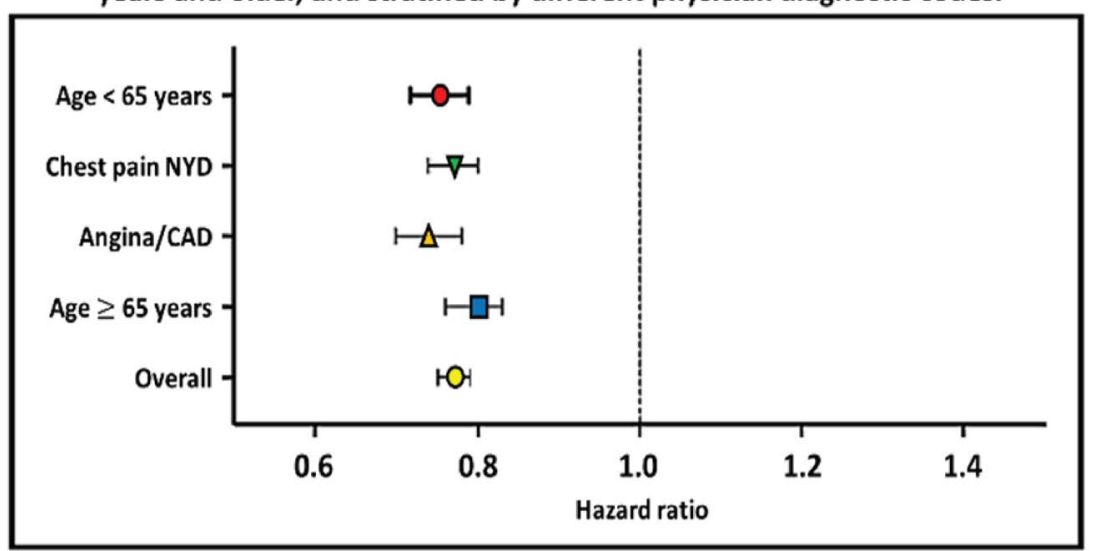

CAD indicates coronary artery disease; NIT, noninvasive

diagnostic tests; and NYD, not yet diagnosed. Roifman I et al. J Am Heart Assoc. 2020;9:e015724.

Many doctors are concerned that we should be rationing healthcare when we use the term parsimonious or 'being stingy'. However, when discussing medical ethics in the context of healthcare policies and costs, Dr Virginia Hood (president of the American College of Physicians clarified that "parsimonious is a good word in the sense that it means that you use only what's necessary," and that she did not "see any particular problems with that. Maybe it has some connotations where people think frugality or being parsimonious is the same as being mean or inadequate."

For resource-poorer nations, more parsimonious, yes, more appropriate, and more cost-effective approaches are called for. Each nation has to weigh its own health efficiencies, as well as its ability to provide easy access, modern infrastructure, sufficient manpower expertise and budget enough allocations to finance its own healthcare system.

more invasive testing or revascularization is some $0.2 \%$ less. More importantly, there appears to be fewer longer-term unstable coronary syndromes and cardiovascular mortality. We await more health audit cost-benefits analyses to see if these would justify advocating more routine cardiac testing in other countries with different socioeconomic bases.

This editorial is to help raise some questions as to the cost vs. benefit balance of what we as cardiologist have been called upon to apply as healthcare providers. We need to scrutinize the extravagance or wasteful use of clinical care algorithms that now besiege the cardiovascular fraternity. We are called upon to exert greater prudence in the use of finite healthcare resources.
Even for richer nations, unfettered wide utilization of health resources can be devastating as these would ultimately lead to reduced access because of inability to pay, including the extremely high costs of insurance, whether through self-paying, employer-benefit or state-paid schemes. ${ }^{9}$

Does a healthcare expenditure of $18 \%$ of the nation's gross domestic product, make sense? ${ }^{10}$ Or can we all work more sensibly and effectively to moderate this escalating cost of diminishing returns? In the field of rapidly more technically advanced management of cardiovascular ailments, cardiologists have to take some lead in moderating the exuberance in healthcare costs escalation.
Many authoritative cardiac societies through its ever more deterministic guidelines and position statements, are redefining and benchmarking ever more diagnostic and therapeutic strategies. These are resultant suppositions of more and more tightly controlled studies that show small incremental benefits, as we strive to be more accurate and precise in our dealing with this nebulous syndrome.

Unfortunately, the wider adoption of such evidence bases has increasingly created demands for greater utilization of costly tests, and innovative devices that severely test the resources of most healthcare systems. Of course, our duty as physicians, is to our patients, whom we wish to manage the best we can. But our patients would ultimately also be those who have to shoulder the mounting cardiovascular health risks and ultimately the healthcare costs for society, as a whole. Thus, we must learn to be more prudent or even be more parsimonious in our clinical decision-making. . $^{6,7}$

\section{Figure 5}

The U.S. Has The Most Expensive Healthcare System

Per capita health expenditure in selected countries in 2018

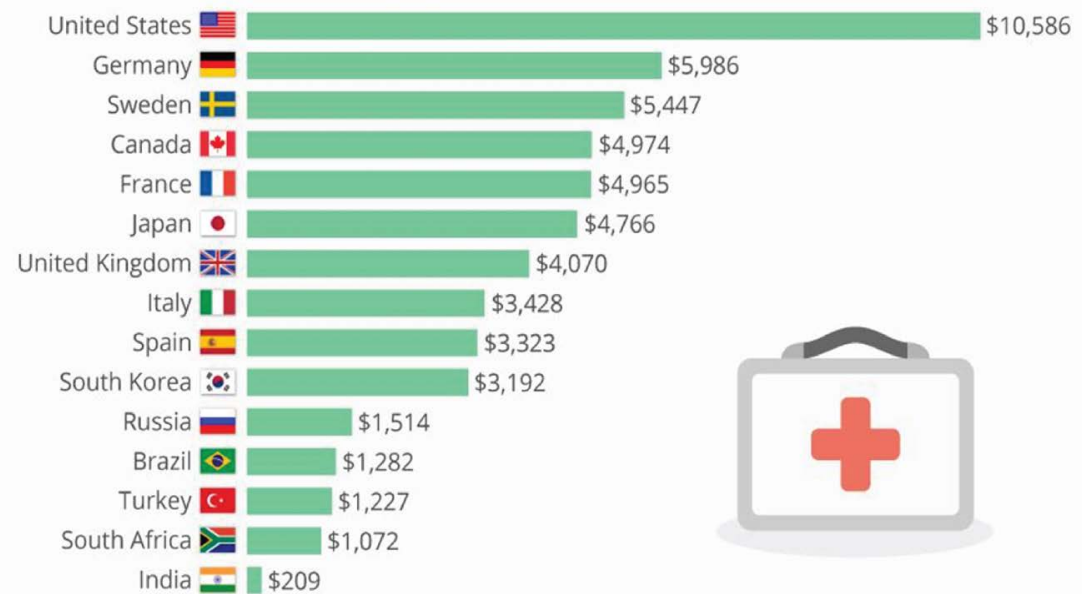


Figure 6: Healthcare Spending vs GDP Growth in the United States

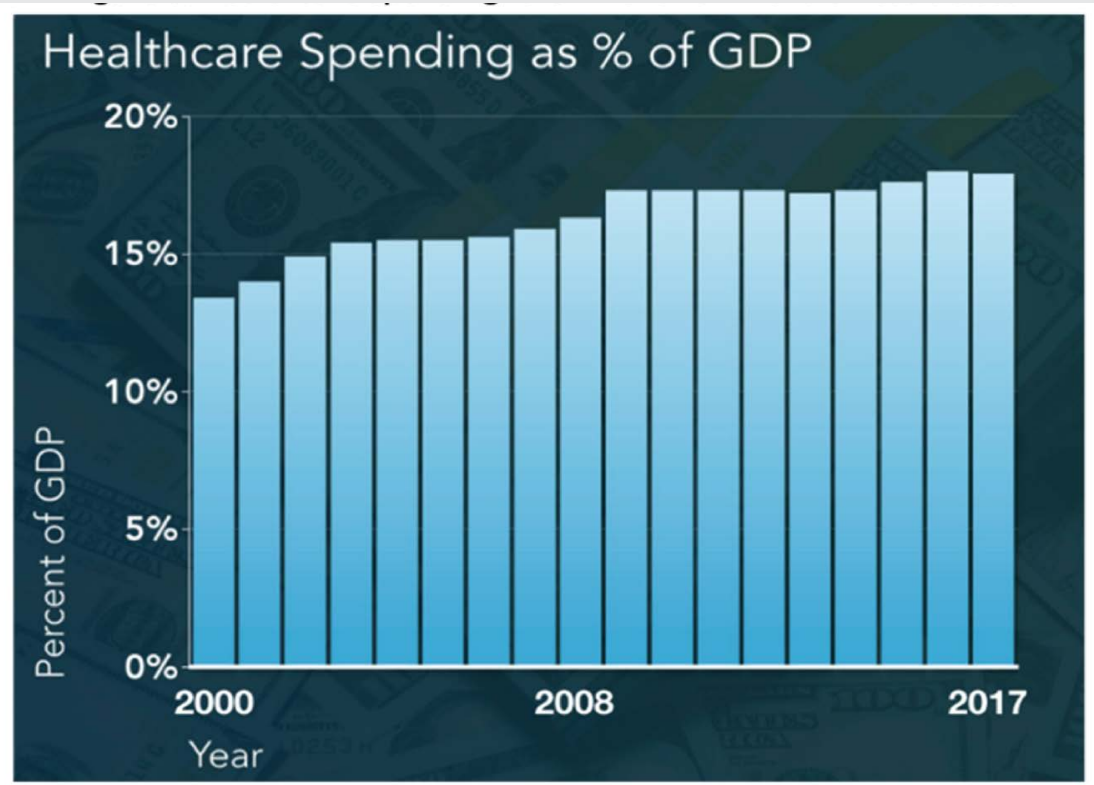

Healthcare spending as a percentage as of the US GDP has plateaued. While healthcare spending continues to grow, the United States GDP has grown at a similar rate. Data Source: National Health Expenditure Accounts, Centers for Medicare \& Medicaid Services

\section{REFERENCES}

1. Juhani Knuuti, William Wijns, Antti Saraste, et al for ESC Scientific Document Group, 2019 ESC Guidelines for the diagnosis and management of chronic coronary syndromes: The Task Force for the diagnosis and management of chronic coronary syndromes of the European Society of Cardiology (ESC). Eur Heart J, 2020;41(3):407-477; https://doi.org/10.1093/eurheartj/ehz425

2. Willis Towers Watson. 2020 Global medical trends survey report. https:// www.willistowerswatson.com/en-US/Insights/2019/11/2020-global-medicaltrends-survey-report

3. Yoshikawa Y, Kimura T. Percutaneous coronary intervention in stable coronary artery disease: still in equipoise? Eur Heart J. 2019;40:187-189.

4. Zimmermann FM, Omerovic E, Fournier S, et al. Fractional flow reserveguided percutaneous coronary intervention vs. medical therapy for patients with stable coronary lesions: meta-analysis of individual patient data. Eur Heart J 2019:40:180-186.

5. Roifman I, Sivaswamy A, Chu A, et al. J Am Heart Assoc. 2020;9:e015724. DOI: 10.1161/JAHA.119.015724
6. Jon C. Tilburt, Christine K.Cassel. Why the Ethics of Parsimonious Medicine Is Not the Ethics of Rationing. JAMA 2013;309(8):773-4.

7. Quek D. Parsimonious Care or prudent rationing in an era of accountability in health care. NHAM Pulse 2013 (March-April):2-8

8. Rob Stein. Should Doctors Be 'Parsimonious' About Health Care? NPR January 3, 2012, https://www.wyso.org/2012-01-03/should-doctors-beparsimonious-about-health-care

9. Niall McCarthy. How U.S. Healthcare Spending per capita compares with other countries (Infographic). Forbes, Aug 8, 2019. https://www. forbes.com/sites/niallmccarthy/2019/08/08/how-us-healthcare-spendingper-capita-compares-with-other-countries-infographic/\#612e80aa575d (accessed 21 Aug 2020)

10. National Health Expenditure Accounts, Centers for Medicare \& Medicaid Services. https://www.cms.gov/research-statistics-dataand-systems/statistics-trends-and-reports/nationalhealthexpenddata/ nationalhealthaccountshistorical 